\title{
PGC Content Production in the Perspective of Digital Labor: Exemplified by Food Uploaders on Bilibili
}

\author{
Ruiyang Qin ${ }^{\text {a }}$, Shubin Zhuang ${ }^{b}$ \\ School of Humanities and Social Sciences, North China Electric Power University, Beijing, China \\ a 120191170423@edu.cn, b120191170301@edu.cn
}

\begin{abstract}
In the post-epidemic era, there are more and more food-related contend on Bilibili. Besides, the purchasing power of Gen-Z is also growing rapidly. Against this backdrop, food brands take Bilibili as their targeted area for promotion to attract young people and build their brand equity. Based on this, the paper chose Bilibili where Gen-Z gather as the study platform and adopted a quantitative research method to analyze the PGC content production. Meanwhile, the paper also selected two major uploaders as the representatives in studying digital laborers' ways to adapt themselves to the era of convergence media, so as to put forward corresponding suggestions. After the analysis, it was found that the current PGC content creators and content production platforms are faced with dilemmas and defects, and how to achieve the production of quality PGC content and get rid of the dilemma of digital labor in content production has become a major concern.
\end{abstract}

Keywords: Digital Labor; PGC Production; Bilibili.

\section{Introduction}

According to the " Bilibili Annual Report on Food Data", in 2021, the view counts of food-related videos played on Bilibili exceeded 24 billion, an increase of $70 \%$ year-on-year, and the view counts of food events video reached 7.2 billion. The food industry has gained more and more attention on Bilibili in recent years. Since Bilibili is quite popular among young people, a lot of food brands have registered their official accounts on it to strive for young potential consumers (especially the Gen-Z). This trend brought both new chances and challenges for the laborers from media. At the same time, with 11 major content categories and 50 secondary subdivision content categories, Bilibili outperforms other video platforms, which marks its high PUGC level. This paper analyzed the content production data of Bilibili's food zone uploaders to study the PGC content production of new media stations in the mode of digital labor, and explored the interaction and co-progression between digital labor and new media platforms.

\section{Literature Review}

Starting from the articles related to the content and with similar purpose of this study, we focused on four aspects (the inner logic of digital labor, PGC content production, the laborization of Bilibili platform and food video uploaders on Bilibili). We have sorted the previous studies in order to determine the research scope and analysis methods and directions.

\subsection{Inner Logic of Digital Labor}

\subsubsection{Research and Value of Studying Digital Labor}

Compared with the long-term accumulation and research base of labor research, the study on digital labor starts much later and the number is relatively small. At the very beginning, when the term "digital labor" was not clearly defined, it often appeared in the study of "audience commodity" under the discipline of political economy of communications. In Communications: Blindspot of Western Marxism, Dallas W. Smythe made such definition, "the commodity form ... under monopoly capitalism is audiences and readerships. ... All non-sleeping time of most of the population is work time. ... Of the off-the-job work time, the largest single block is the time of audiences which is sold to advertisers. Here come the demand management. In the process of production, selling, and 
consumption, audience power becomes a valuable commodity."[1] According to Wang's view shown in How Does "Labor" Become Communication Tools? --- A Paradigm Shift and Theoretical Logic from "Audience Commodity" to "Digital Labor", the "audience commodity" theory is mainly based on television as a communication medium and focuses on the self-fulfillment mechanism of the media industry at a macro level. Audiences' attention becomes a kind of labor when watching advertisements, and the commodity demand is generated through the payment of attention. [2]

In the Digital Labor and Karl Marx, Fuchs made a relatively clear definition for digital labor:" ... digital labor ... are part of a collective work force that is required for the existence, usage and application of digital media. What defines them is not a common type of occupation, but rather the industry they contribute to and in which capital exploits them. " [3] Accordingly, this paper argued that in this era when everyone can speak for himself, digital laborers have the dual identity of media platforms. They can be passive recipients of information and at the same time, they can actively choose to receive or reject the content of information, and produce and disseminate information by themselves. As a result, the users are motivated by a new force and gain new interpersonal relationship, contributing their free labor to capital. In the process of creating labor, the additional consumption behavior deepens capital's exploitation of the audience's surplus value.

\subsubsection{Classification of Digital Labor}

In Wu's Laborlization of Internet Audience: On Status of Internet Audience in Media Industry from the Perspective of Political Economy of Communication and Yao's The Dilemma and Measures of Digital Labor in the Gig Economy, digital labor can be divided into three categories in terms of its working form. The first category is the production and consumption of content on interactive social platforms. Users are to some extent contributing their collective wisdom and collective labor without compensation to form the platform atmosphere and platform image. The second category is the professional users under the flexible employment system. The third category is the playbours in the commercial industry chain. Julian Kücklich put forward the term "playbour" quite early, and defined it as users who create value in their leisure time by playing [4] [5]. Many of the digital laborers work for the new media platforms that produce content based on PGC and UGC modes. Users' secondary creation is empowered and enabled by new media technology, and some new media users have developed into free laborers who are willing and able to produce and disseminate content. As for this paper, the third type mentioned about is taken as the target group to analyze their content generated and the commercial operation.

\subsubsection{Problems of Digital Labor}

From the definition and classification listed above, it is clear that the prevalence of "digital labor" reflects the neglection of the value of human subjectivity, and users are aware of the existence of labor exploitation The weak emotional relation and little value return of the online platform alone are no longer able to keep the users. According to the current research on digital labor, the problems of digital labor mainly exist in two aspects: labor intensity imbalance and labor-management relationship. Li Mengming, in Understanding the Labor Guidance of We-Media Platforms for Users from the Perspective of "Digital Labor", discussed the time span the audience can control, which highlights that digital laborers currently suffer from a general imbalance of outputs and inputs and the work-life boundaries are not clear enough. In particular, the labor time of PGC and UGC users does not bring an equivalent increase in corresponding benefits. Besides, Ding Yiran talked about the exploitation mechanism in Breaking Through From "Exploitation": The Status Quo, Problems, and Re-defamiliarization of Digital Labor Research and pointed out that the current studies available on exploitation of digital labor are simplistic and homogenized, unable to universally explain the exploitation in diverse labor and lacks process analysis of the mechanisms. 


\subsection{New Business State and Operation of New Media Platforms}

\subsubsection{Novel Path for Communication Media}

In CMG Video "PGC+PUGC"Mode Innovation and Mainstream Value Leading, Long and Pan found that the Internet video platforms are in the third stage of its development. Due to the defects in the quality of UGC content, high-quality video content is required. A large number of users with professional content production skills entered into the mobile video platform and become content creaters. Such a fusion of UGC and PGC content production account has become the platforms' hot cake.

\subsubsection{Platform Characteristics of Bilibili and the Operation Logic}

Lu, in her work Study on the Interaction Mechanism between PGC Short Video Creation and Embodied Cognition, argued that The major new media platforms are now characterized by a combination of general content and professional ones, visualization of text, personalization of promotion, fragmentation of consumption and interaction within groups. [6] In Long and Pan's study on the innovation mode of CMG, they stated that The ecological value of the combination of specialized and generalized content is in line with the fragmented learning characteristics, broad learning interests and high-quality learning needs of users in the Internet era. [7] According to Zhang's view presented in Reconstruction of Content Production Model under the Perspective of PGC-Take CCTV's "Live for Finance" as an Example, we can get similar results in Bilibili [8]. Bilibili's panknowledge section collects content with high quality produced by PGC and PUGC accounts, which can be specially promoted. Compared with UGC content production without content standards and professionalism, PGC content production relies more on teams and expertise with the more systematic and standardized production. The combination of specialized production and the new media discourse model promotes the specialization and diversification of content production on new media platforms. Meanwhile, in "Exploring the Popularization Path of Group Culture - Analysis of Bilibili's 2019 Annual New Year's Eve Celebration", Fu and Lin also proposed one of Bilibili's operation mindset "cultural integration ". "The core driving force of Bilibili's subculture gaining more attention is the cultural innovation with more inclusive environment. Bilibili is not confined to its own cultural resources, Instead, it tore its label for a single cultural group and try to embrace more groups.[9] The inclusiveness and its popularity make Bilibili's effort in reaching cultural recognition more prominent. $\mathrm{Qu}$ and Zhang also stressed that Bilibili's success is closely related to the "philosophy of discontinuity" in Fracture and Continuity of Culture in the Internet Era: A Study of the Prevalence of Work on Traditional Themes on Bilibili. [10]

\subsubsection{Uploaders' Self-development under New Business State and Operation}

Wu and Jiang also mentioned in their paper "The Network Practice of Silver-Haired Netizens and their Pursuit" that the homogenization of the current network bloggers is very serious. [11] Wu and Jiang also mentioned in their paper "The Network Practice of Silver-Haired Netizens and their Pursuit" that the homogenization of the current network bloggers is very serious. In the post-epidemic era, the content of Bilibili's food section has ushered in an explosive period of growth. As Gen-Z's purchasing power has gradually become stronger, the growth of food brands has been driven by the entry into the young market of Bilibili's "food" category. The industry has seen a large number of uploaders with high quality who can create popular videos.

It was found through the video data of Bilibili food section in the past year that the highest monthly output video reached 270,000 in 2021, and the total play counts and the number of pop-up comments were about 2 times of the number of videos in food industry, and the total number of coins invested was more than the average level of the whole industry. Undoubtedly, the food-related content gained more attention and ranked $1^{\text {st }}$ in terms of Internet traffic volume. Even so, the homogeneity of uploaders is extremely obvious. The food uploaders can be divided as food store visiters, folk cuisine, gourmands and food producers. Even with 42 food sub-catergories, the videos of the same kind basically follow similar video shooting methods and segments. Due to the unstandardized platform 
guidance and capital system, the gourmands have been criticized by the mainstream media due to safety issues and negative publicity. Therefore, this article will also discuss how the food category uploaders in such an environment will find a way out.

The above studies related to digital labor content production and new media platforms reveal that although the focus is different, the analysis framework and perspectives can be summarized into three aspects: the internal logic and dilemma of digital labor, the characteristics of the new business model of new media platforms, and the innovation of the communication model of new media platforms.

In summary, this paper took the example of the food content uploaders of Bilibili as an example, and explained the use of PGC production in new media platforms like Bilibili from the perspective of the inner logic of digital labor, content production and commercial operation of the new media platform. It provided new ideas for the current research on the interaction between digital labor and new media platforms.

\section{Research Design}

\subsection{Research Methodology and Sample Selection}

This study adopted a quantitative content analysis method, using Bilibili, a platform with a widespread PGC model and a strong community culture, as the main research platform. Two uploaders of the most popular categories of food,@肉肉大搜索 and@别叫我狗哥, who entered the category at the earliest stage, were selected because of their vlog styles (known for food store visit and food production respectively). @ 肉肉大搜索 joined Bilibili in 2020, and is one of the earliest food explorer releasing vlog on Bilibili. He has got more than 2.29 million followers, 16.42 million likes and 420 million plays. For @ 别叫我狗哥, he was introduced to Bilibili after he rose to fame on Douyin. Most of his video are about food making and his roommates' daily life(especially for what they eat everyday). He has acquired 719,000 followers, received 11,021,000 likes and had 160 million plays.

\subsection{Categories and Fields}

After sorting out the data related to Bilibili food category uploaders, the hotlist and video statistics, the study selected the food store visit field and the food production field as the representatives. Two leading uploaders were selected as an example (one for each). They are @肉肉大搜索 and @ 别叫 我狗哥. In the recent quarter from October 2021 to January 17, 2022, @肉肉大搜索 and @别叫我 狗哥 cumulatively released 20 and 91 videos respectively, with fan increments of 322,000 and 141,900. This paper, starting from the main body of uploaders, followers and the platform respectively, by referring to the framework of previous studies and the characteristics of PGC content output, analyzed the content of their videos from the following seven criteria.

(1) Frequency and Duration

(2) Types of food - snack, Chinese food, Western food, beverage, desser

(3) Performance - effect of the video and coverage

(4) Hot words - keywords and characteristics

(5) Video style - background music, editing style, image presentation

(6) Interaction - time and form (Q\&A, self-deprecation, offer proposals, daily life sharing and product introduction)

(7) Followers image - gender, the account of VIP followers and their characteristics

(8) Degree of the prevalence - keywords of the repost and comment 


\section{Analysis on PGC Content Creation from the Angle of Digital Labor}

\subsection{Basic Condition}

\subsubsection{Video Number and Release Frequency}

This part is about the number of the video released in the past 90 days and the frequency.

(1) Number of new videos released

@ 别叫我狗哥 posted 91 videos in 90 days, basically reaching a daily frequency, while @ 肉肉 大搜索 posted 20 videos in 90 days. There is a large gap between the two. It is mainly because of the limitation of the video content style, as @肉肉大搜索, a food store explorer, needs to choose the location and visit the store, while the video content of @别叫我狗哥 are more about the daily food making, so he is less restricted by the time and place.

\section{(2) Video Duration \& Update Frequency:}

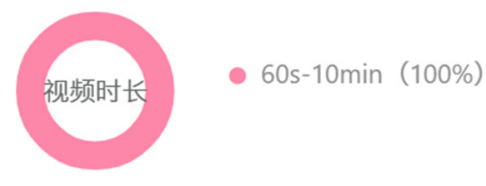

(@肉肉大搜索)

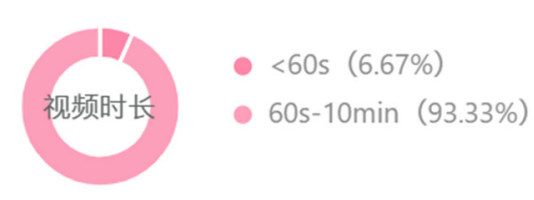

(@别叫我狗哥)

Fig 1. Video Duration \& Update Frequency

@ 别叫我狗哥maintains a daily update with a relatively short video (about 7\% of the videos within 60s and most of them are based on life), whereas@肉肉大搜索 updates relatively slowly (three times a week) with a relatively large video volume, whose content is business-oriented.

The major users of Bilibili, an important media platform, are highly sticky fan groups and Generation Zs. Both of the two popular bloggers focus on food, maintaining a relatively stable daily or weekly output, which serves as an operation tool to keep followers loyal and accounts active. Limited by the subject, the update frequency of A @肉肉大搜索 relatively slow, but still maintains stable. As a new food blogger, @ 别叫我狗哥 uses daily update to quickly attract traffic and improve the popularity on TikTok and Blibili.

\section{(3)Update Time:}

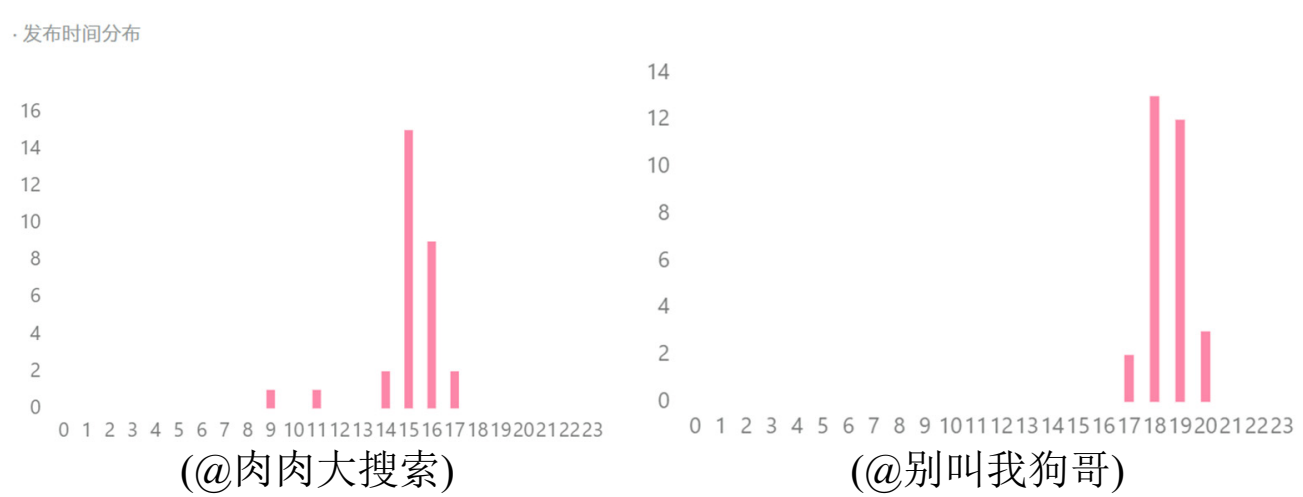

Fig 2. Update Time

As shown from the distribution, the updates of @ 肉肉大搜索 are relatively scattered, mainly ranging from 15:00 to 16:00, with the most frequent releases being at 15:00, while the videos released 
by @ 别叫我狗哥 are concentrated at a time period between 18:00 and 19:00, with 18:00 being the most popular posting time point.

The most frequent posting time is 15:00 and 18:00 (both in the afternoon). The two fixed updating times are concluded based on the previous posting patterns of the two bloggers.

\subsubsection{Video Type}

(1)Food Type:

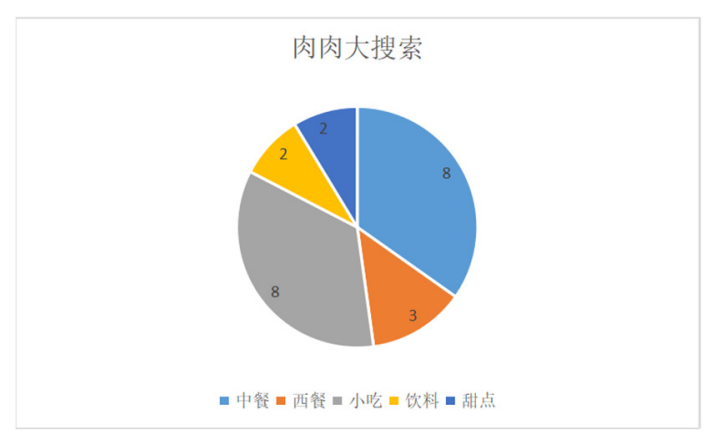

(@肉肉大搜索)

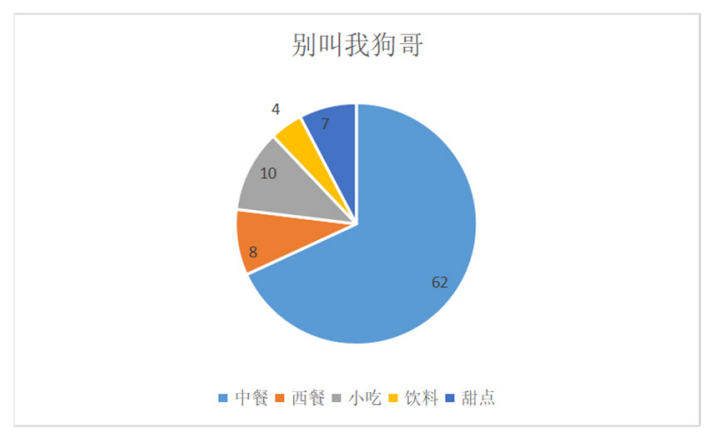

(@别叫我狗哥)

Fig 3. Food Type

From the perspective of food type, 80\% of the videos of @肉肉大搜索 focus on snacks and Chinese cuisines. Compared with@别叫我狗哥, the food types of @肉肉大搜索 are relatively balanced and rich. However, @别名我狗哥 focuses more on Chinese cuisines, which accounts for about $70 \%$ of the videos, mainly influenced by the home-cooked food. The reason why the two bloggers present different food types to enrich the video content is mainly because a single food type cannot attract the fan groups with diversified needs.

(2)Video Style:

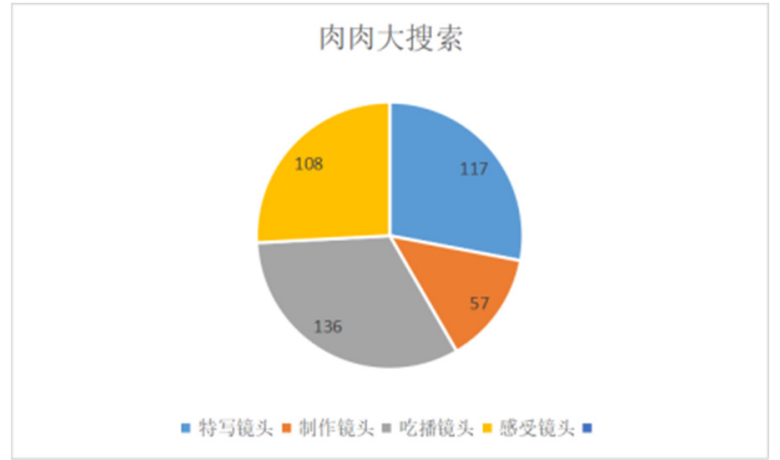

(@肉肉大搜索)

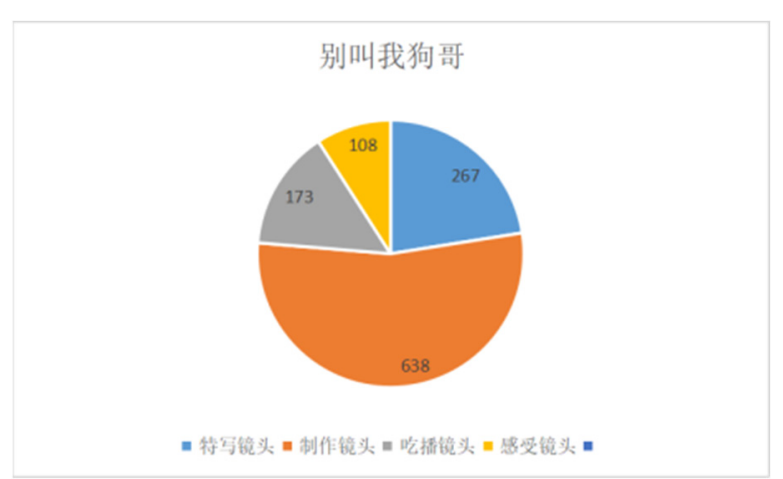

(@别叫我狗哥)

Fig 4. Video Style

In general, the videos of the two bloggers are completely different in tone. @肉肉大搜索 favors mature commercial food videos with good camera use. The videos of @肉肉大搜索 are mainly made up of eating and taste sharing, which accounts for more than 60\% of the shots, while @ 别叫我 狗哥 has basically 7 production scenes in 91 videos, in line with the characteristics of its food vlog.

In terms of music use, the back ground music of @ 肉肉大搜索 is more harmonious and relaxing, whereas@别叫我狗哥 has more funny sound effects, which are more lifelike. 


\subsubsection{Performance in Different Sectors}

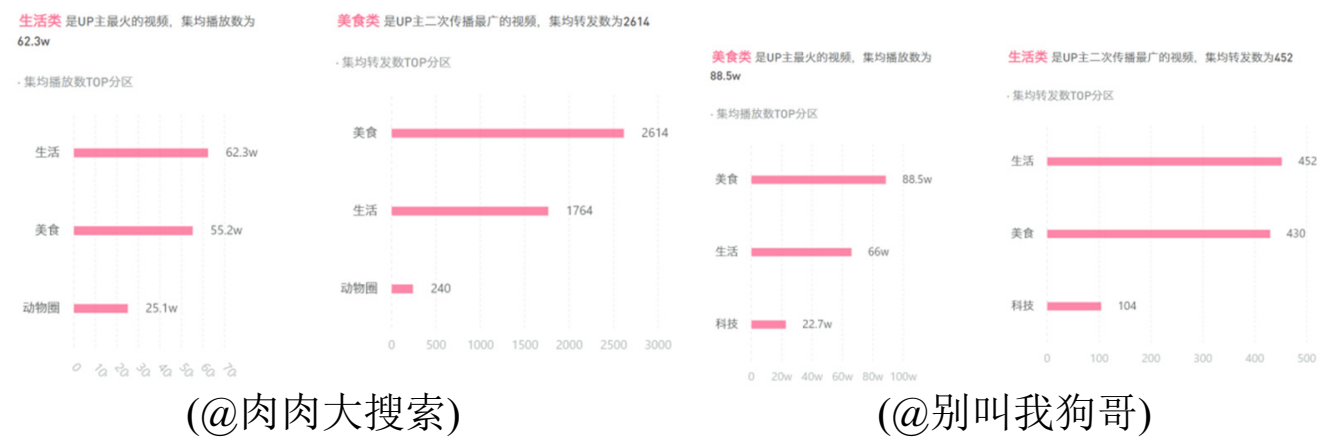

Fig 5. Performance in Different Sectors

The significant difference between the audience groups of the two uploaders can be seen from the above data. The video of @肉肉大搜索 is more widely spread in the food area, with an average repost number reaching 2614 per episode, but is more popular in the daily life area. The video of @ 别叫我狗哥 is more widely spread in the daily life area, and is popular in the food area, with an average play number reaching $885 \mathrm{k}$ per episode.

That has a significant relationship with the video style and video theme of the two bloggers. Bilibili features bullet chatting culture. The high quality bullet chats and the real-time interaction of the videos also increase the entertainment. @肉肉大搜索 explores the national cuisine, providing a wider platform for the bullet chatting culture. Meanwhile, the maturity and interaction of his videos are higher than that of @ 别叫我狗哥, so they have more obvious advantages in the spread of the food area and the popularity of the daily life area.

\subsubsection{Video Popularity}

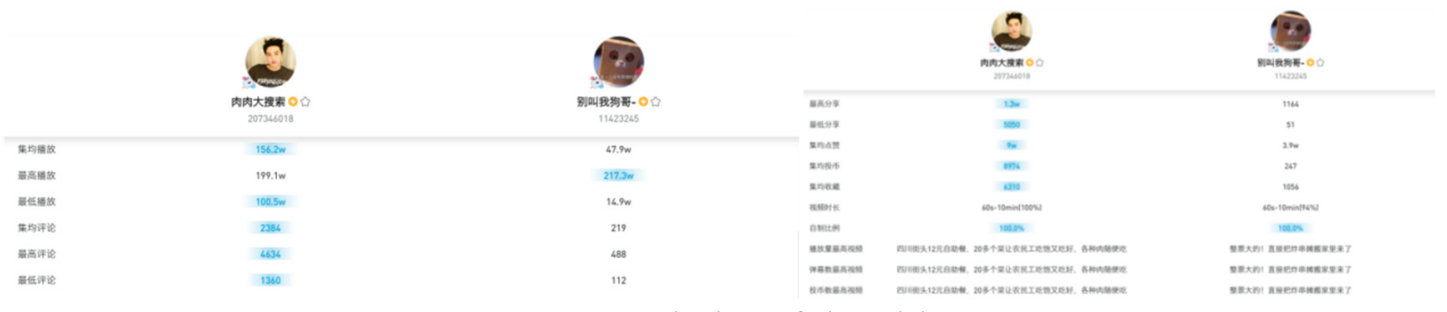

Fig 6. Popularity of the video

As the above data shows, the data of @肉肉大搜索 is generally better than that of @ 别叫我狗 哥, except the highest play counts (@肉肉大搜索 1991k, @别叫我狗哥2173k). The two bloggers' likes and reposts all exceed 10 thousand, but the comments are relatively less. The comments number of @ 别叫我狗哥 is less than a thousand. In terms of advertising, both bloggers have placed a small number of ads, but it can still be seen that the videos have less interaction and popularity once the content or theme involves advertising. Thus, the videos released with advertising should be limited in a proper number. The videos with the highest popularity of both bloggers are related to the life with high emotional value. The hottest video of @ 肉肉大搜索 is related to the plight of migrant workers, while the most popular video of @ 别叫我狗哥 reflects the daily life of a food stall, both of which are associated with users' emotional value.

\subsection{Analysis of Followers' Preference}

In conducting the follower profile analysis, obvious differences in the fan groups were found, which are attributed to the obvious differences in the video style, the dissemination period, and the accumulated popularity. 


\subsubsection{Followers Increase}

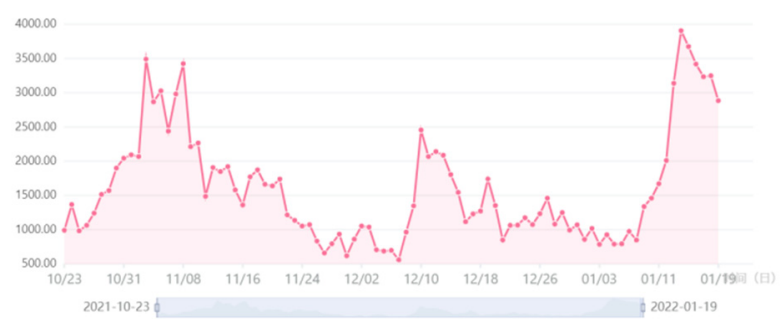

(@别叫我狗哥)

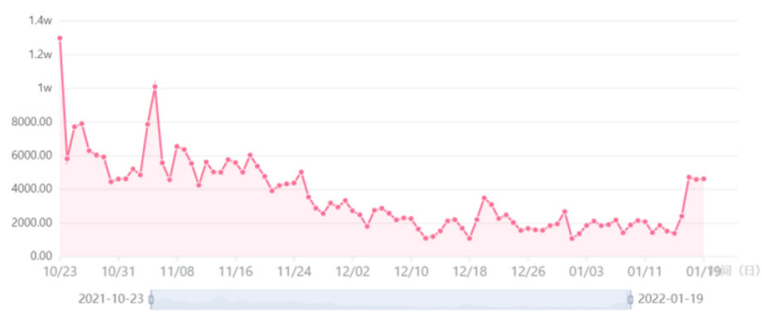

(@)肉肉大搜索)

Fig 7. Followers Increase

From the above data, it is obvious that in the past 90 days, the daily increase of @ 别叫我狗哥's followers has reached 4,000 with an average daily increase staying around 1,500, while that of@肉 肉大搜索 has reached 13k with an average daily increase remaining around 3,000. There is a huge gap between the two, but based on the followers number, the increase keeps in a reasonable range. At the same time, it can also be seen that after the emergence of @ 肉肉大搜索's hot video on October 23 , the fan growth achieved a great change, which shows the significant influence of high quality works for bloggers to attract traffic.

\subsubsection{Buzzwords in Videos}

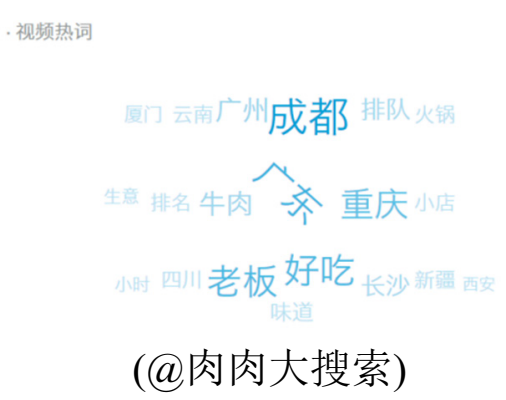

Fig 8. Buzzwords in Videos

As the hot words in bullet chats show, although the two bloggers belong to food sector,@肉肉大 搜索 focuses more on food, such as delicious, cheep, pork intestines, pork tripe, beef, etc., whereas @ 别叫我狗哥 is more related to daily life, such as mom, pajamas, the same items, warm, etc.

\section{Discussion on PGC Content Production from the Perspective of Digital Labor}

\subsection{Value of Pgc Content Production Model}

\subsubsection{The Combination of "Special" and "General" Content Production in New Media}

As a Gen Z video platform, Bilibili is important for food brands and food bloggers. As one of the earliest popular categories, "food" enjoys a leading position in terms of traffic and audience, and a large number of high-quality bloggers and popular videos are emerging in the industry.

To make the platform ecology diversified, Bilibili covers hundreds of first- and second-level categories including knowledge, music, sports, and entertainment, realizing the comprehensiveness and richness of the pan-cultural platform. Meanwhile, taking the food sector as an example, with a deep understanding of Gen $\mathrm{Z}$ consumption view, the food sector of Bilibili is divided into 42 food channels to further refine the category circles. The emerging categories develop fast and remain today, for instance, Li Ziqi, the representative of garden food, Mianyang cuisine, the representative of home cooking, Rishiji, the representative of one-person food. @ 别叫我狗哥 is catering to this trend, who 
transformed from pet life sharing to cooking for roommates and then to home cooking, becoming a typical blogger who updates on both TikTok and Bilibili. After being settled in Bilibili, its video volume has increased significantly, and the content also becomes more specialized and richer. In contrast, UGC short video platforms such as TikTok, Kuaishou, are rich in content, but the quality is spotty and homogeneous. Users generally spend a lot of time viewing short videos to get a seemingly large amount of information, but finally the effective information memorized is very limited.

\subsubsection{Diversified Video Forms: Long and Short, Shallow and Meaningful}

At present, the short video is flourishing, whereas the long video, which is a product of traditional media and traditional media derivatives, is gradually shrinking in scale, and even some categories are disappearing. However, we still have to realize that any kind of media has its own value, and the newly emerging media can only play a complementary role instead of completely replacing the old one. According to Paul Levinson's Media Evolution Theory, when a new medium outperforms the old one in a particular field, it does not mean that the old medium is about to decline and die, but in fact, the old medium is forced into a smaller living environment in which it survives because of its own uniqueness.

At present, under the strong impact of TikTok, Kuaishou, Weishi and so on, the three traditional video giants Youku, Aqiyi, and Tencent, are subject to the limitations of long video, facing an uncertain future. Bilibili, as a following video website, has created a "short plus long" video pattern. Moreover, it builds a special video section including dramas and films to maintain the long video ecology. The famous bullet chatting community culture of Bilbili not only provides a free and open platform for film and drama clips, which makes it easy to create hot topics, attracting traffic from short to long videos, but also offers a broad stage for the discussion of quality dramas and films. Apart from that, the "short plus long" effect is also significant in other areas. For example, in the food sector, @ 别叫我狗哥 updates very frequently, and the short videos play a role in attracting traffic, which help its long videos of over 10 minutes to be viewed regularly.

\subsection{The Soft Value Implantation of Pgc Content Production}

Traditional video texts reflect the compulsory discourse comprehension and meaning interpretation mode of elite culture, and the video content tends to be commercialized, which uses intelligent algorithms to collage, integrate and recombine texts, sounds and images in a patterned way, thus stimulating the audience's senses and interest and maximizing the traffic, topics, and values. However, as audiences themselves become laborized, the video content is no longer top-to-bottom in communication, but turns to be interactive, so the patterned commercial content output can no longer meet the needs of the audience. The dual identity of labor's content output and reception requires its own emotional needs attached to the creation. From the keywords such as "mom" appear in @ 别叫 我狗哥's comments, it can be seen that emotional value is added in @ 别叫我狗哥's creation to trigger the viewers' empathy.

In terms of PGC video creation, content producers, whether they are organizations or individuals, have their own cognition and aesthetics of the world, which makes the video output more diversified and personalized. As the popular videos of @肉肉大搜索 and @ 别叫我狗哥 show, the emotional value plays a major role in traffic. The video "12 yuan buffet in Sichuan: 20 dishes with meat enough make migrant workers eat well" of @肉肉大搜索 makes a huge contrast in headline to attract users' curiosity. Meanwhile, it combines the hot topics of people's livelihood with humanistic care from the business perspective to arouse the audience's emotion.

\subsection{Difficulties and Countermeasures of Digital Labor in Pgc Content Production}

With the development of the integrated media era, the boundaries of digital labor have become clearer. In the media era where almost all people participate, digital laborers have a dual identity, being both the information recipients and the content producers on media platforms. However, this has led to the problem of imbalance of output and input and ambiguity of work-life boundaries among 
digital laborers. In particular, PGC content producers keep outputting quality content but the return is disproportionate.

The paper believes that there are two main reasons for this phenomenon: First, the intangible benefits. The emotional and spiritual satisfaction brought to users by new media platforms cannot be measured by standard data. Second, free participation. Users' participation in new media creation is often unrestricted, which further leads users to ignore the time boundary between creation and leisure. It can be seen that @ 别叫我狗哥 keeps daily creation, with 91 food videos in 90 days. Behind such update frequency is the team's daily efforts in theme generation, script development, food creation, food filming, and post-editing. Even if the video content is relatively daily, it takes considerable effort. The revenue gained from such creation with no advertising on Bilibili is minimal, and the number of coins invested is only $17 \mathrm{k}$ at present.

\subsubsection{Countermeasures for Digital Labor Facing Pgc Content Production Difficulties}

\subsubsection{The Construction of the Platform Incentive System}

With the continuous shock of the short video platform, a number of old Bilibili bloggers leave the platform. Such results are led by the lack of long-term revenue from content production in the digital labor area. In 2017, the Oasis Program was launched to build a bridge for bloggers and brands to gain advertising exposure. Also in August 2018, the "High Power Alliance" program was introduced. In addition, there are commercial measures such as incentive programs, charging programs, and reward programs.

In the absence of brand advertising revenue, Bilibili users have to fully cater to the platform policies and adjust their content to participate in various incentive activities to make money. Because the interests of the creators are linked to the platform share, PGC content producers and the platform are to a certain extent interest bundles. The platform achieves labor control through incentive policies to guide the creators to produce content that meets the expectations and tones of the platform. However, this also creates another dilemma, the output quality will be affected under the capital interference, which leads to another way of content output profitability.

\subsubsection{The Maximization of Fan-oriented Interests}

Hochschild once pointed out that in addition to the laborer's body, the emotions are also the object of capital management. Apart from the platform's comment section and bullet chatting interaction design, the content output of digital labor is a kind of emotional connection outside the platform. A healthy interaction between creators and their followers will bring a greater traffic.

Besides the emotional maintenance initiatives of the official platform, there is another layer of emotional relationship beyond the platform, the emotional interaction between creators and followers, which can accumulate more followers and traffic and improve their influence. When the content is good enough and has a certain amount of traffic, the platform's incentive policy will no longer be the most important component of labor earnings. They can profit from commercial advertising and fan coins, getting rid of the platform's labor exploitation.

\section{References}

[1] Smythe, D. Communications: Blindspot of Western Marxism[M].1977.

[2] Wang, J.H. How Does "Labor" Become Communication Tools? --A Paradigm Shift and Theoretical Logic from "Audience Commodity" to "Digital Labor" [J]. Journalism and Mass Communication Monthly, 2018 (10): 56-64.

[3] Fuchs, C. Digital Labor and Karl Marx [M]. China Social Sciences Press.2016.

[4] Wu, D.M. Laborlization of Internet Audience: On Status of Internet Audience in Media Industry from the Perspective of Political Economy of Communication [J]. Chinese Journal of Journalism \& Communication. 2017(6):124-137. 
[5] Yao, J.H. The Dilemma and Measures of Digital Labor in the Gig Economy [J]. Contemporary Communication. 2018 (3): 66-68.

[6] Lu, J. Study on the Interaction Mechanism between PGC Short Video Creation and Embodied Cognition [J]. Journal of Hunan Administration Institute.2021(4):103-109.

[7] Long, Y. \& Pan, X.T. CMG Video "PGC+PUGC" Mode Innovation and Mainstream Value Leading [J]. TV Research.2021(4):22-25.

[8] Zhang, L. Reconstruction of Content Production Model under the Perspective of PGC - Take CCTV's "Live for Finance" as an Example [J]. China Radio \& TV Academic Journal.2021(6):107-109.

[9] Fu, X.G. Exploring the Popularization Path of Group Culture - Analysis of Bilibili's 2019 Annual New Year's Eve Celebration [J]. News and Writing.2020(2):86-90.

[10] Qu, C.J. \& Zhang, T.Y. Fracture and Continuity of Culture in the Internet Era: A Study of the Prevalence of Work on Traditional Themes on Bilibili. [J]. Modern Communication.2018(9):86-92.

[11] Wu, Y.H. \&Jiang, Y. Network Practice of Silver-Haired Netizens and their Pursuit. [J]. News and Writing. $2021(3): 14-21$. 